\title{
Localized Knowledge based System for Human Disease Diagnosis
}

\author{
Adane Nega Tarekegn \\ Faculty of Computing, Bahir Dar University,Ethiopia \\ E-mail:nega2002@gmail.com
}

\begin{abstract}
Knowledge based system can be designed to solve complex medical problems. It incorporates the expert's knowledge that has been coded into facts, rules, heuristics and procedures. Incorporation of local languages with the knowledge based system allows endusers communicate with the system in a simpler and easier way. In this study a localized knowledge based system is developed for TB disease diagnosis using Ethiopian national language. To develop the localized knowledge based system, tacit knowledge is acquired from domain experts using interviewing techniques and explicit knowledge is captured from documented sources using relevant documents analysis method. Then the acquired knowledge is modeled using decision tree structure that represents concepts and procedures involved in diagnosis of disease. Production rules are used to represent domain knowledge. The localized knowledge based system is developed using SWI Prolog version 6.4.1 programming language. Prolog supports natural language processing feature to localize the system. As a result, the system is implemented using Amharic language (the national language of Ethiopia) user interface. With Localization, users at remote areas and users who are not good in foreign languages are benefited enormously. The system is tested and evaluated to ensure that whether the performance of the system is accurate and the system is usable by physicians and patients. The average performance of the localized knowledge based system has registered $81.5 \%$.
\end{abstract}

Index Terms-Knowledge Based system, Localization of KBS, Disease diagnosis, Amharic Language user interface, artificial intelligence.

\section{INTRODUCTION}

The excess morbidity and mortality in developing countries is caused by communicable diseases [22]. Tuberculosis (TB) is one of the of complex human communicable diseases and leading cause of morbidity and mortality in Ethiopia. Co-infection with HIV leads to challenges in both the diagnosis and treatment of tuberculosis. Further, unavailability and scarcity of medical experts in health centers are the major problems in Ethiopia. In an effort to address such problems, this study focuses on designing a localized knowledge based system for TB diagnosis and treatment.

Knowledge based systems are assisting physicians in making decision without consulting the specialists directly [1, 6]. In medical diagnosis, knowledge based systems are mainly used for performing diagnoses based on patient data, since they can naturally represent the way experts reason. According to $[1,2]$, the main inspiration of developing knowledge based system includes: to provide many (electronic) copies of an expert's knowledge, to preserve expertise knowledge for future use and to distribute experts' knowledge in a structured manner. One major issue along with the distribution and preservation of expert's knowledge is the Localization issues. Distribution and preservation of experts' knowledge using local languages could have profound benefits [3].

Knowledge Based System Localization is demanding research area in the field of natural language processing. Localization involves developing knowledge based systems as per local and cultural needs and translation of the user interface and other system terminologies into the local languages [4]. The system is developed with Amharic language user interface. In the knowledge based system, the tasks of a natural language interface is to translate user statements into facts of the underlying knowledge based system.

Advantages of local language user interface $[4,5]$ are the following:

- The domain specificity of most knowledge based systems provides strong constraints on the vocabulary, actions and concepts that may be expressed in natural language.

- User is not forced to learn an artificial communication language.

- No special training is required before using the natural language interface. It is highly user friendly and easy to use by the end users.

- The natural language interface in very simple and easy to use because the end users write the query in their native language.

\section{Methodology OF THE STUdY}

\subsection{Literature Review}

There are different studies on knowledge based system which have been done in the medical domain. Aderonke et al [1] developed an integrated knowledge based system for Histopathological Diagnosis of Breast Diseases. This 
work was developed to simulate the expertise of a pathologist that leads to a proper diagnosis of breast disease. Solomon [10] proposed a self-learning knowledge based system for diagnosis and treatment of diabetes. This study was conducted using rule based reasoning technique that can provide advice for physicians and patients in order to facilitate the diagnosis and treatment of diabetic patients.

Soundraraja and Sureshkumar [19] proposed diagnostics decision support system for tuberculosis. This research was done using fuzzy logic representation technique to find the probable class of tuberculosis a patient may have. In [20] we find fuzzy cluster means expert system for the diagnosis of tuberculosis. This study was limited to only extra pulmonary TB and uses neuro solutions and crystal reports for neural network analysis and graphical representation. Seblewongel [21] presented a rule-based system for anxiety mental disorder diagnosis. This study was conducted to find solutions for mental health problem. We also find another related work in [23] an intelligent medical system for diagnosis of common disease that can be detected by patient data.

However, as to the researcher's knowledge no attempt has been done to design a knowledge based system for diagnosis and treatment of tuberculosis. Moreover, most of the knowledge based systems didn't support local languages and also didn't propose a treatment method for clinical activity. Moreover, the previous literatures didn't demonstrate user acceptance testing of the system which is done by users to ensure that whether the proposed system satisfies the requirements of its end-users. Thus, in this study an attempt is made to design a knowledge based system that can support local language and provide the corresponding treatments that the system can diagnose.

\subsection{Data Collection Method}

The data source for this study was FelegeHiwot Referal Hospital, Bahir Dar, Ethiopia. There are many different kinds of complex human disease diagnosed in the hospital. For this study, the researcher selected tuberculosis (TB) disease which is one of the most complex and deadliest diseases in the world. During data collection, explicit and tacit knowledge are acquired from both documented sources and non-documented sources respectively. Non-documented sources of knowledge are acquired from internists and medical doctors who work in the FelegeHiwot Hospital by using interviews and discussions. Similarly, documented sources of knowledge such as medical books, training manuals and journal medical articles are acquired by using document analysis technique. In addition to tacit and explicit knowledge, documented tuberculosis patient history is collected from patient records for evaluation purpose.

\subsection{Sampling Techniques}

Purposive sampling technique is used to select domain experts for knowledge acquisition and to collect previous TB patient cases archived in FelegeHiwot Hospital. The selection criteria of domain experts for the study is based on the profession or expertise, educational qualification level, year of experience and their immediate position in the TB disease diagnosis. Five experts have been consulted in the courses of study and one BSc nurse is involved in the registration of the patient cases from the card collection. Purposive sampling is one of the most common sampling techniques in qualitative research in which participants are decided preselected criteria relevant to a particular research question [7]. Expert sampling is a type of purposive sampling technique that is used when the research needs to gather knowledge from individuals that have particular expertise. It is also called judgmental sampling that occurs when the researcher interviews a panel of individuals known to be an expert in a given domain. Expertise can be any special knowledge, not necessarily formal training, depending on the topic of study [8].

\subsection{Knowledge Modeling and Representation}

In this study, after the knowledge is extracted from documentary and non-documentary sources, it is modeled using decision tree structure. Decision trees models by constructing a tree based on training instances with leaves having class labels is used. These are easy to interpret and can be represented as if-then rules. The knowledge acquired from different sources has to be represented using the knowledge representation methods. After the acquired knowledge is modeled, it is represented by using knowledge representation methods. Production rules are the most popular form of knowledge representation methods for knowledge based system development. Knowledge is represented in the form of condition/action pairs: IF this condition occurs, THEN some action (or conclusion) will (or should) occur [1]. Production rules are also easy for a human expert to read, understand and maintain. Production rules contain simple syntax which is flexible and easy to understand and are reasonably efficient in diagnosing of human diseases. The reasons for using productions rules in this study are because of its ease of modifications and applicability when there is a need to recommend a course of action based on observable events [9].

\subsection{Implementation Tool}

Prolog programming language is used to develop the localized knowledge based system. The reason for selecting this programming language is the features and abilities of the language that incorporate it. Prolog is a declarative language and has the capacity to describe the real world. Because of its declarative semantics, built-in search, and pattern matching, Prolog provides an important tool for programs that process natural language. We can write natural language grammars directly in Prolog.

\section{KNOWLEDGE ACQUISITION}

Knowledge acquisition is the process of collecting, extracting, structuring and organizing knowledge, and 
recording it in a convenient form for representation from different sources like human experts, books, medical documents, journals and the Internet, so it can be used in knowledge-based system development [11]. This is usually done through visual, aural, reading and mining from large documents in which a knowledge engineer receives through different techniques. The acquisition of knowledge is a major and critical phase in the development of knowledge based systems and is still the most difficult and error-prone task that knowledge engineer does while building a knowledge-based system.

\subsection{Explicit Knowledge Acquisition}

There are several sources of explicit knowledge including literatures, books and guidelines. The knowledge captured here should be used as a reference and to confirm the tacit knowledge captured from the human expert and should never replace an experienced domain expert. The following are some sources for explicit knowledge acquisition [12]: Literatures, Reports, guidelines, Published books and journal articles.

\subsection{Tacit knowledge Acquisition}

Since printed materials can sometimes become outdated and not sequentially arranged, printed materials should never be considered as sufficient sources of information for the development of knowledge-based systems. The experience that guides the reasoning of a domain expert cannot all be found in printed sources such as books. The range of problems which a textbook examines and solves is always smaller than the range of problems that a human expert is master of [12]. So, Acquiring domain knowledge from the human expert is needed for development of knowledge based system. The most important part of knowledge acquisition is knowledge elicitation which consists of a set of techniques and methods that attempt to elicit the knowledge of a domain expert, typically through some form of direct interaction with the expert [13].

\subsection{Knowledge Acquisition Process}

Knowledge elicitation was performed through Knowledge Acquisition. In order to acquire required knowledge for this study both secondary and primary sources of knowledge are used. Secondary source of knowledge involves gathering knowledge from existing documentations. The primary sources of knowledge include TB experts who work at FelelegeHiwot hospital, Bahir Dar, Ethiopia. During the preliminary investigations, seven experts from FelelegeHiwot hospital have been contacted to understand the dimension of TB disease. During this time, the researcher is trying to conduct informal kinds of interviews with these experts. However, for extensive discussion with experts, the researcher selects five experts. The experts were purposively selected for the interview by their knowledge of TB and years of experience they worked on TB. These experts are consulted throughout the research work to evaluate the correctness of the acquired knowledge and to verify the cases acquired from the previous patient history.

To collect the knowledge from a given specific domain there are different techniques available such as interview, protocol analysis, document reviews, questionnaires and card analysis [36] which can be used to acquire both tacit and explicit knowledge. Basically the researcher used structured interview to acquire knowledge of domain experts and organizational document analysis to acquire knowledge from relevant documents. This is because of a structured interview is a systematic, goal oriented process It forces organized communication between the knowledge engineer and the expert.

\section{KNOWLEDGE TRANSLATION}

In localization of the knowledge based system, translation of the domain knowledge is the primary task as it is a crucial component of localization. Translation is the process of actually converting the written word of a source language into the written word of a target language. In the localized knowledge based system, the user interface and all related words are translated into Amharic language with the help of domain experts. In addition to the domain experts, the researcher used the following resources to translate English documents of TB into Amharic language:

- $\quad$ TB HIV merged participant's manual in Amharic for health extension workers:-This is a training document for TB in Amharic language for health extension workers prepared by Ethiopian Federal Ministry of Health with support from development partners.

- Minnesota department of health (http://www.health.state.mn.us/tb): it is an online resource that contains TB documents in different languages such as in Amharic, Arabic and other.

\section{KNOWLEDGE MODELING}

Conceptual modeling is considered to be an important stage in knowledge based system development process. According to Pamela [14] conceptual modeling is the activity of formally describing aspects of the domain knowledge for the purposes of understanding and communication. The knowledge engineer has to determine what aspects of the real world to include and exclude from the model. The way that this is done depends on the needs of the potential users, the domain to be modeled and the objectives to be achieved. There are many techniques used for knowledge modeling such as decision tree, semantic network, UML, and hierarchy of frames. For knowledge-based systems, decision trees have the advantage of being comprehensible by domain experts and of being directly convertible into production rules i.e. decision tree has the ability to represent the problem in natural and simple if-then description [15]. Moreover, when used to handle a given case, a decision 
tree not only provides the solution for that case, but also states the reasons behind its choice. Thus decision tree is used in this study to model the elicited domain knowledge.

\subsection{Modeling Pulmonary TB Diagnosis}

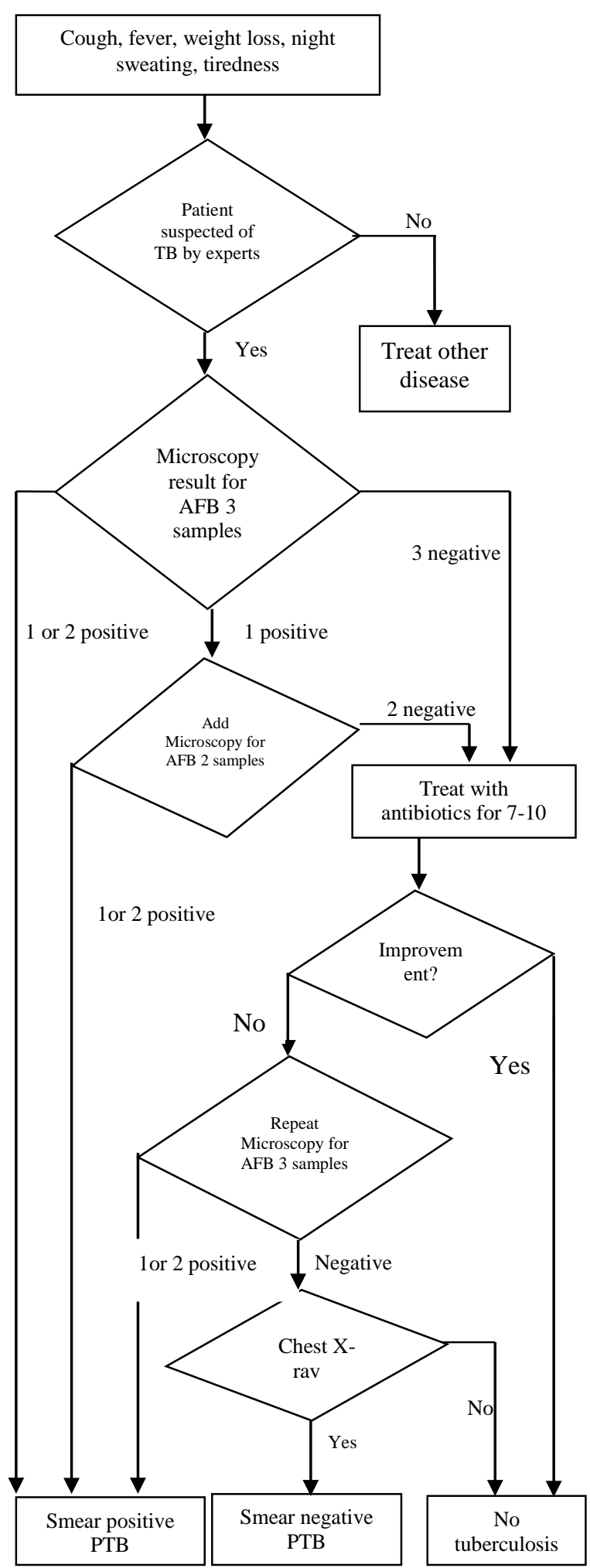

Fig.1. Decision tree for Pulmonary TB
In the diagnosis process, any person who presents with symptoms or signs suggestive of $\mathrm{TB}$, in particular cough of more than 2 weeks is a TB suspect and should have a sputum examination. Starting sputum examination quickly helps the patient to start the treatment as early as possible for better outcome and to interrupt the chain of transmission of TB.

For diagnostic purposes, all persons suspected of having TB disease at any site should have sputum specimens collected for an AFB smear. At least three consecutive sputum specimens are needed, each collected in 8 to 24 hour intervals, with at least one being an early morning specimen. Three sputum specimens must be examined by smear microscopy.

The decision tree based on conceptual model depicted in figure 1 shows the levels of decisions that domain experts use during diagnosis. It is modeled by classifying the laboratory smear result as three positive (3+), two positive $(2+)$, one positive $(1+)$ or all negative.

\section{KNOWLEDGE REPRESENTATION}

After the raw data is acquired from the domain experts, it must be modeled and converted into intermediate representations. Knowledge representation is the process of encoding domain knowledge in to machine understandable representation. In this study production rules are used to represent the domain knowledge. Rules for the knowledge based system are constructed based on the knowledge modeled using decision trees. The following are fragments of sample rules of the knowledge base in their English like translation.

If patient has symptom of TB

Then the patient is TB suspect AND must check sputum microscopic test for AFB

If sputum microscopy test result for $\mathrm{AFB}=2$ positive or 3 positive from the three samples

Then the Patient has smear positive pulmonary TB

AND treat for smear positive pulmonary TB

If sputum microscopy test result for $\mathrm{AFB}=1$ positive from the three sample

Then Check for additional sputum microscopic test for AFB

AND sputum result $=1$ positive or 2 positive

Then patient $=$ smear positive pulmonary $\mathrm{TB}$

OR Sputum microscopic test result $=2$ negative

AND antibiotics for 7-10 days

AND Improvement after 2-4 weeks?

Yes $=$ No TB

$\mathrm{No}=$ check sputum

If Sputum result $=1$ or 2 or 3 positive

Then patient $=$ smear positive pulmonary $\mathrm{TB}$

If Sputum result $=3$ negative

Then Check chest X-ray of the patient

If chest X-ray $=$ suggestive of TB

Then patient $=$ smear negative pulmonary TB OR

If Chest X-ray = normal

Then patient has No Tuberculosis, check for other disease. 


\section{IMPLEMENTATION}

The localized knowledge-based system is implemented based on the decision tree model and the knowledge represented in a form of rule-based representation. The SWI Prolog implementation tool is used to develop the prototype, which has different components. It involves user interface, knowledge base, and inference engine and explanation facility. Figure 2 shows the architecture of the system which is constructed.

As shown in Figure 2, the user enters symptoms of the TB disease and/or the laboratory results into the localized KBS in Amharic language. The facts and rules of the knowledge base are accessed to make a diagnosis. If the system gives diagnosis to the problem, it is displayed to the user and the details of a specific patient diagnosis or treatment results are stored in the knowledge base of the localized system for future use.

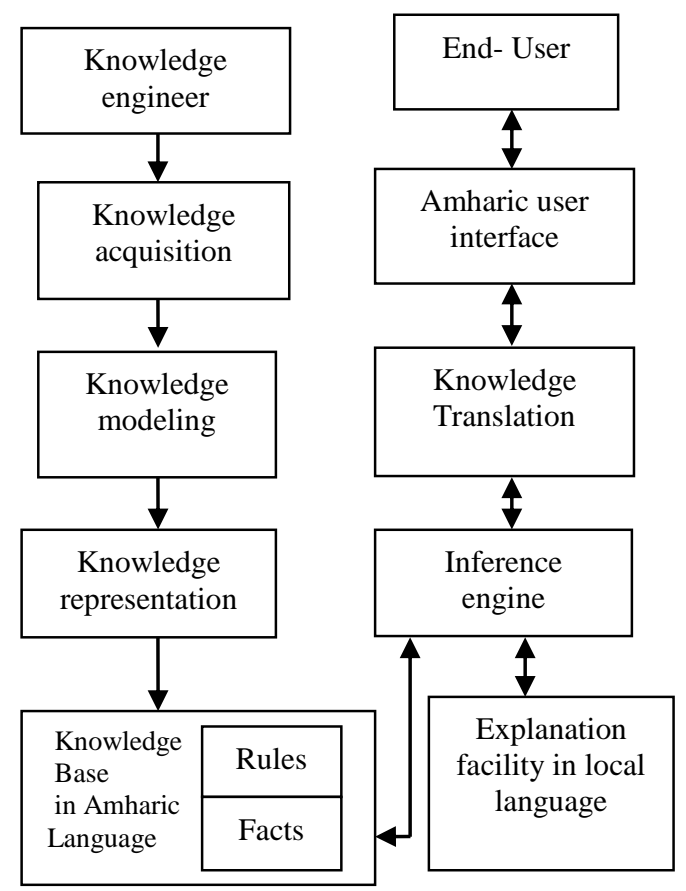

Fig.2. Architecture of the knowledge based system

\section{STARTING THE LOCALIZED SYSTEM}

The end-users of the system can start and use the system by simply double clicking on file 'kbs.pl' which stored on the local user machine. Then the welcome window will be displayed automatically as shown in figure 3 .

After the welcome window is displayed automatically, the system requests if the end-user wants to continue using the system. If the user wants to continue using the system, he/she must type the Amharic word "中rA." and the system continues displaying the next step with instructions for the user. It displays the menu which contains different choices using Amharic language such

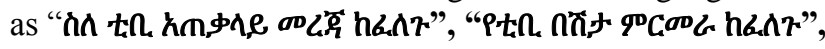

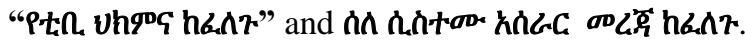

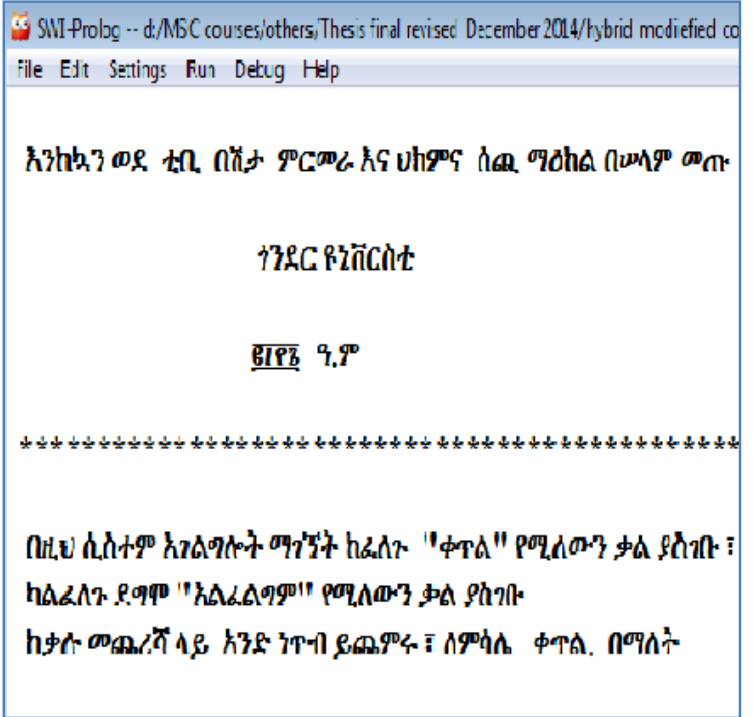

Fig.3. Welcome screen of the localized KBS

\begin{tabular}{|c|}
\hline Q6 SWI-Prolog - d/MSC courses/others/Thesis final revised_December 2014/hyteri \\
\hline File Edit Settings Run Debug Help \\
\hline 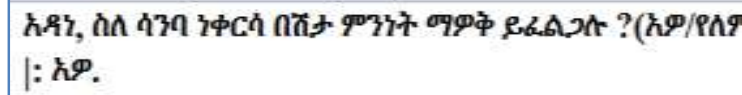 \\
\hline 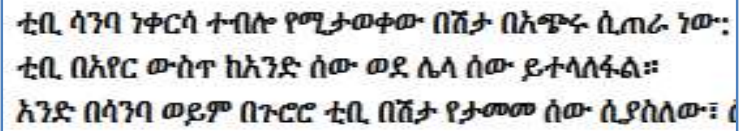 \\
\hline 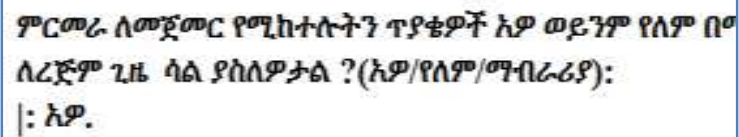 \\
\hline 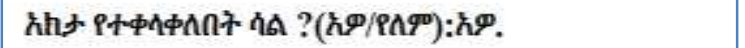 \\
\hline 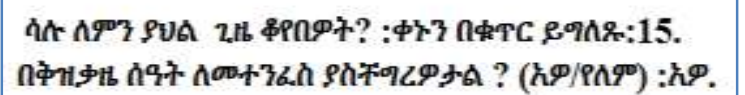 \\
\hline 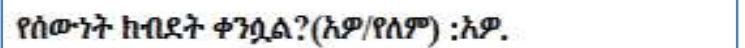 \\
\hline 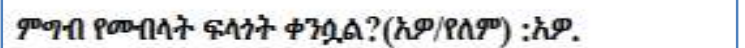 \\
\hline
\end{tabular}

Fig.4. User interaction with the localized KBS

Figure 4 shows the interaction of a system with the end-users. The system displays different questions to the user. The user answers each of the questions with ' $\mathbf{h} \boldsymbol{\Phi}$ ' or 'PAqD' which is yes or no in English respectively.

\section{TESTING AND Evaluation}

Evaluation is an important issue for every knowledge based systems. It is a technique used to demonstrate the performance of the system and its acceptance by the end users. The purpose of the evaluation process is to get the 
end user's views on the significance or usefulness of the system. The evaluation and testing issue of the system answers the question "To what extent the localized knowledge based system give acceptable and accurate diagnosis and treatment service to tuberculosis patients?" To answer this question, system performance testing and user acceptance testing methods are used.

\subsection{User Acceptance Testing}

User acceptance testing is the process of ensuring that whether the system satisfies the requirements of its endusers. This allows users of the system to test the prototype system by actually using it and evaluating the benefits received from its use. A questionnaire is prepared to evaluate the user acceptance of a system, and the evaluators fill the questionnaire after they have used the system. The questionnaire prepared is used to measure the system performance based on the standard of user acceptance validation. The researcher adopted the questions from Audrey Mbogho [16], Solomon [10] and Ashenafi [17]. Table 1 represents the questions that users used to evaluate the system.

Table 1. Questions for user acceptance testing

\begin{tabular}{|l|l|}
\hline $\begin{array}{l}\text { Questions } \\
\text { number }\end{array}$ & Questions \\
\hline Question 1 & The system is simple to use during interaction? \\
\hline Question 2 & $\begin{array}{l}\text { the results and descriptions provided by the } \\
\text { system are easily understandable }\end{array}$ \\
\hline Question 3 & $\begin{array}{l}\text { The system operates in a very good speed and } \\
\text { efficiency }\end{array}$ \\
\hline Question 4 & $\begin{array}{l}\text { the system is helpful and } \\
\text { free physicians from boring routine tasks }\end{array}$ \\
\hline Question 5 & Coverage of TB knowledge is sufficient \\
\hline Question 6 & $\begin{array}{l}\text { the commands are easy to learn and understand } \\
\text { for new users }\end{array}$ \\
\hline Question 7 & $\begin{array}{l}\text { Use of Amharic language increases the } \\
\text { simplicity and understandability of the system }\end{array}$ \\
\hline Question 8 & $\begin{array}{l}\text { The advice provided by the system is accurate } \\
\text { and useful }\end{array}$ \\
\hline
\end{tabular}

The evaluators fill in the questionnaire as strongly agree, agree, neutral, disagree and strongly disagree for each of the questions. The author assigned values for each word or phrase as strongly agree $=5$, agree $=4$, neutral $=3$, disagree $=2$ and strongly disagree $=1$. A total of six domain experts are participated in the system evaluation. Table 2 shows the evaluation results.

Table 2. Results of Evaluation

\begin{tabular}{|l|l|l|l|l|l|l|}
\hline $\begin{array}{l}\text { Que } \\
\text { stion }\end{array}$ & $\begin{array}{c}\text { Strongly } \\
\text { Agree }\end{array}$ & Agree & $\begin{array}{c}\text { Neut } \\
\text { ral }\end{array}$ & $\begin{array}{c}\text { Disag } \\
\text { ree }\end{array}$ & $\begin{array}{l}\text { Strongly } \\
\text { Disagree }\end{array}$ & Average \\
\hline 1 & 2 & 3 & 0 & 1 & 0 & 4.00 \\
\hline 2 & 2 & 2 & 1 & 1 & 0 & 3.83 \\
\hline 3 & 3 & 2 & 1 & 0 & 0 & 4.33 \\
\hline 4 & 3 & 2 & 1 & 0 & 0 & 4.33 \\
\hline 5 & 2 & 1 & 3 & 0 & 0 & 3.83 \\
\hline 6 & 1 & 3 & 2 & 0 & 0 & 3.83 \\
\hline 7 & 3 & 2 & 0 & 1 & 0 & 4.16 \\
\hline 8 & 3 & 2 & 0 & 1 & 0 & 4.16 \\
\hline & \multicolumn{6}{|l}{ Total Average } \\
\hline
\end{tabular}

It is found in Table 2 that most respondents agree that a user can use and interact with system easily. The simplicity of the system showed $83.33 \%$ scored as agreement, $16.67 \%$ as disagreement. Similarly, $83.33 \%$ of the respondents agreed with the speed of the system as it is fast but $16.67 \%$ of them remained neutral.

For evaluation criteria 4 , it was found that most users agree that the system can be very helpful and could reduce some of the workload for medical assistants especially during peak times $(50 \%$ of them selected strongly agree, $33.33 \%$ agree and $16.67 \%$ remained neutral). This will decrease the long queues in clinics because other physicians can still use the system without assistance. For evaluation criteria 5, it was found that most of the respondents agreed that the system has incorporated sufficient knowledge of the domain which scores about $50 \%$ and $50 \%$ of them argued that the system didn't incorporated sufficient knowledge of the domain.

For evaluation criteria 7 , the system uses local language to communicate with the intended users. So, the users have also given their opinion on the use of Amharic language during interaction. Based the collected results around $33.3 \%$ of the respondents are strongly agreed on the use of Amharic language to increases the adoption of local conventions and understandability of the system, $33.33 \%$ of them grouped as agree and the remaining $33.33 \%$ as disagree. The total sums of the users that take the advantage of Amharic language in the system are about $66.66 \%$. This indicates that the majority of the users agreed on the incorporation of Amharic language in the system. However, the remaining $33.33 \%$ of the evaluators didn't agree with the use of Amharic language in the system because of that most of the medical terms didn't have equivalent Amharic meaning and are unusual and difficult to understand sometimes.

Generally, it was found that most evaluators have positive feedback about the localized KBS, especially about the knowledge that system contains. The average performance of the prototype system according to the evaluation results filled by the domain experts is 4.05 out of 5 or $81 \%$ which shows the users are satisfied with the localized knowledge based system.

\subsection{System Performance Testing}

This testing method is applied to evaluate the performance of the knowledge based system using the parameters precision, recall and F-measure. These three parameters are used in order to measure the accuracy of the prototype system. In the process of testing the performance of the KBS, the domain experts classify correctly and incorrectly diagnosed patients cases by comparing the judgments reached by the KBS system with that of the domain experts ${ }^{\text {ee }}$ judgments reached on the same patients' test cases.

Performance of this system is usually evaluated using the data in the matrix. Table 3 shows the confusion matrix for a three class classifier.

A confusion matrix is a visualization tool typically used to present the results attained by the learner. Each 
column of the matrix represents the instances in a predicted class, while each row represents the instances in an actual class. One benefit of a confusion matrix is that it is easy to see if the system is confusing two classes. In Table 3 above, of the 8 actual smear positive pulmonary TB patients, the system predicted that 2 were smear negative pulmonary TB patient, and of the 8 smear negative pulmonary TB patients, it predicted that three were smear positive pulmonary TB. We can see from the matrix that the localized system can make the distinction among smear positive pulmonary TB; smear negative pulmonary TB and extra pulmonary TB pretty well.

Table 3. Confusion matrix of the localized KBS

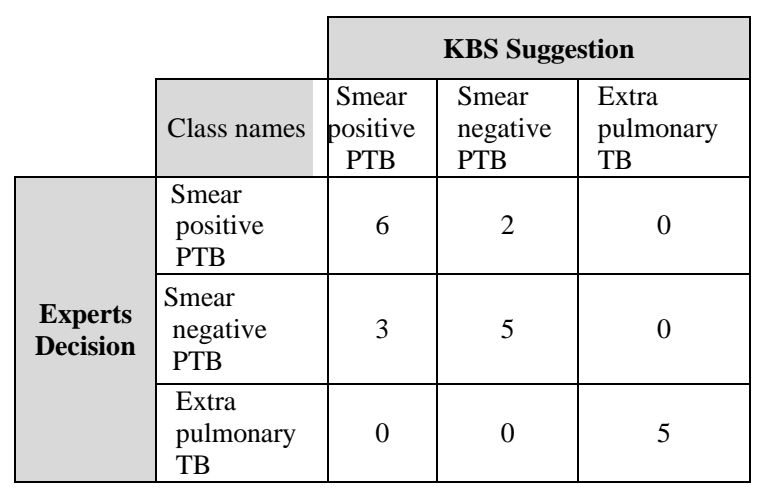

From Table 3, we can see that the result of the testing has revealed $82 \%$ accuracy in diagnosing TB cases. The detailed generated result for this performance measure is shown in table 4 which includes the precision $(\mathrm{P})$, recall(R) and F-measure.

Table 4.Accuracy of the localized KBS

\begin{tabular}{|l|l|l|l|}
\hline \multirow{2}{*}{} & Recall & Precision & Class names \\
\cline { 2 - 4 } & 0.67 & 0.75 & $\begin{array}{l}\text { Smear positive } \\
\text { pulmonary TB }\end{array}$ \\
\cline { 2 - 4 } & 0.62 & 0.71 & $\begin{array}{l}\text { Smear negative } \\
\text { pulmonary TB }\end{array}$ \\
\cline { 2 - 4 } & 1.00 & 1.00 & Extra pulmonary TB \\
\hline $\begin{array}{l}\text { Average } \\
\text { Weight }\end{array}$ & $\mathbf{0 . 7 6}$ & $\mathbf{0 . 8 2}$ & \\
\hline
\end{tabular}

As shown in Table 4, the precision for extra pulmonary TB scores the highest rate $(100 \%)$ followed by smear positive pulmonary $\mathrm{TB}(75 \%)$ and smear negative pulmonary TB (71\%). The recall for extra pulmonary TB is $100 \%$ which is the highest score. The weighted average precision is $82 \%$ which shows the KBS system has an accuracy of $82 \%$ in diagnosing TB patient cases, that is, of the $21 \mathrm{~TB}$ cases, $82 \%$ of the cases are diagnosed correctly. Similarly, the weighted average recall is $76 \%$ which shows the ability of the KBS system to select instances from a set of cases.

\section{Results AND Discussion}

In this study both system performance testing and user acceptance testing have been done for the localized knowledge based system. As indicated in section 9, the accuracy of the system is calculated as $82 \%$. In addition to accuracy, user acceptance evaluation of the system has been calculated as $81 \%$. The average performance of the localized knowledge based system is $81.5 \%$ which is an encouraging result.

The researcher has faced some challenges during the study which limits the knowledge based system to register a better performance for diagnosis and treatment of tuberculosis. These are discussed as follows:

- During the system development process, it was difficult for the researcher to convert all the needed knowledge into the local language due to that translating medical knowledge into Amharic language was a challenging task.

- The performance of the localized system depends directly on the quality of the knowledge acquired from domain experts. However, knowledge elicitation from domain experts was the most difficult task due to that tacit knowledge is difficult to transfer to another person by means of writing it down.

\section{CONCLUSION AND FUTURE WORK}

Knowledge based system is basically an interactive computer programme that can help a great deal in disease diagnosis and treatment. In this research, a localized knowledge based system that supports diagnosis of TB disease was developed. The aim of integrating local languages to knowledge based systems is to provide an interface where user can interact with the expert system more easily using their local language. The system is a localized knowledge based system that contains Amharic language user interface. The Amharic user interface can help end-users to understand the diagnosis more easily and to distribute information in their native language.

The system is evaluated using different evaluation methods and achieved $81.5 \%$ of an average performance. The localization techniques used in the knowledge based system can be taken as strong features of the system. However, the system lacks to update rules in the knowledge base of the system and the user interface need to be enhanced with a better graphical user interface that allows users to choose their language preferences. So, further study is needed to improve user interface of KBS systems and to design a system that can update rules of the knowledge base. 


\section{REFERENCES}

[1] Aderonke A. et al. "An Integrated Knowledge Base System Architecture for Histopathological Diagnosis of Breast Diseases". I.J. Information Technology and Computer Science, PP.74-84, V.01, 2013.

[2] Audrey Mbogho, "Knowledge Based Expert System for Medical Advice provision". MSc thesis, department of Computer Science, University of Cape Town, 2012.

[3] Jaime, Michael L., Peter G. "A Natural Language Interface to Expert Systems". Unpublished, Computer Science Department, Carnegie Mellon University, 1985.

[4] Varsha Tomar, Manisha Bhatia. "Localization of Text Editor using Java Programming", International Journal of Computer Applications, Vol.89, No.12, PP.49-54, march 2014.

[5] Jaime, Michael L., Peter G. "A Natural Language Interface to Expert Systems". Unpublished, Computer Science Department, Carnegie Mellon University, 1985.

[6] Koushal, Gour Sundar. "Advanced Applications of Neural Networks and Artificial Intelligence: A Review". I.J. Information Technology and Computer Science, V.7, PP.57-68, 2012.

[7] Mack, N., Kathleen, C., Macqueen, M., Guest, G. and Namey, E. "Qualitative research methods: A data collector field guide". Internet: http://www.fhi360.org/resource/qualitative-researchmethods-data-collectors-field-guide, July 2011, [March, 2014].

[8] G. D. Garson, "Sampling," 2012 Edition ed: Statistical Association Publishing, 2012.

[9] Pearson Education. "Chapter 18 - Knowledge Acquisition, Representation, and Reasoning", URL: http://wps.prenhall.com/wps/media/objects/3778/3869053 /Turban_Online_Chapter_W18.pdf, [Feb 02, 2014].

[10] Solomon A. "a self-learning KBS for diagnosis and treatment of diabetes." MSc Thesis, Addis Ababa University School of Information Science, Ethiopia, 2013.

[11] Ashenafi chalchissa. "Developing a knowledge-based system for counseling students at ethiopian higher education institutions". MSc thesis, University of Gondar, Department of computer science, Ethiopia, 2013.

[12] Rhem, A.J and Associate inc. "Frame work of knowledge acquisition".

Internet: http://webcache.googleusercontent.com/search?q=cache: 1 BwBc5AQmjwJ:www.ajrhem.com/framework.pdf $+\& c d=$ $1 \& \mathrm{hl}=\mathrm{en} \& \mathrm{ct}=\mathrm{clnk} \& \mathrm{gl}=\mathrm{et}$, May 2011[April 2014].

[13] Nigel R. Shadbolt and Paul R. Smart." Knowledge Elicitation: Methods, Tools and Techniques". Internet: http://www.amazon.co.uk/Evaluation-Human-WorkFourth-Wilson/dp/1466559616/, 2014].

[14] Pamela Faber. "Conceptual modeling in specialized knowledge resources". Unpublished, University of Granada, Spain, 2009.

[15] Hussein A, Shigeo K., Yasuhiro. "Development and applications of decision trees". unpublished, japan, 2002.

[16] Audrey Mbogho, "Knowledge Based Expert System for Medical Advice provision". MSc thesis, department of Computer Science, University of Cape Town, 2012.

[17] Ashenafi chalchissa. "Developing a knowledge-based system for counseling students at ethiopian higher education institutions". MSc thesis, University of Gondar, Department of computer science, Ethiopia, 2013.

[18] Abdullah, M.S., Kimble, C., Benest, I. and Paige, R. "Knowledge Based Systems: a re - evaluation". Journal of Knowledge Management, Vol. 10, No. 3, Pp. 127-142,
2006

[19] K.Soundararajan, Dr.S.Sureshkumar, C.Anusuya." Diagnostics Decision Support System for Tuberculosis using Fuzzy Logic". International Journal of Computer Science and Information Technology \& Security (IJCSITS), Vol. 2, No.3, June 2012.

[20] Imianvan, A. A., and J. C. Obi. "Fuzzy cluster means expert system for the diagnosis of Tuberculosis." Global Journal of Computer Science and Technology, Volume 11 Issue 6, pp.41-48, April 2011.

[21] SebleWongel."Developing prototype knowledge-based system for anxiety mental disorder diagnosis". MSc Thesis, Addis Ababa University School of Information Science, Ethiopia, 2012.

[22] World health organization. "Global tuberculosis report 2013":

URL:http://www.who.int/tb/publications/global_report/en /, May 2012.

[23] Dipanwita, et al. "Disease Diagnosis System". URL: http"//www.interscience.in,2014.

\section{Author's Profile}

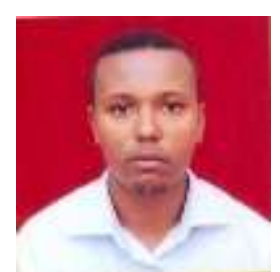

Mr. Adane Nega is currently working as a lecturer at the faculty of computing, Bahir Dar University, Ethiopia. $\mathrm{He}$ obtained his M.S.c degree in information technology from university of Gondar and B.S.C degree in computer science from Bahir Dar University, Ethiopia. His area of research interests include Artificial intelligence and soft computing, Data mining, big data analysis, machine learning.

How to cite this paper: Adane Nega Tarekegn,"Localized Knowledge based System for Human Disease Diagnosis", International Journal of Information Technology and Computer Science(IJITCS), Vol.8, No.3, pp.43-50, 2016. DOI: 10.5815/ijitcs.2016.03.05 